\title{
Calidad en la administración de medicamentos intravenosos y su efecto en la seguridad del paciente
}

\author{
Quality in the administration of intravenous drugs \\ and its effect on patient safety
}

Mtra. Patricia Adriana Cárdenas Sánchez*, Mtra. Rosa Amarilis Zárate Grajales**.

* Enfermera Especialista "C" del Hospital Infantil de México Federico Gómez. ** Profesor de Carrera Asociado "C". T.C. ENEO - UNAM.

\section{Resumen}

El propósito de este estudio es evaluar la calidad en la administración de medicamentos por vía intravenosa que proporciona el profesional de enfermería a través de la aplicación de un instrumento que mide el índice de eficiencia, lo que permitirá determinar las fallas o casi-fallas e implementar estrategias de mejora, pero también identificar sus fortalezas para reforzarlas. Además los resultados, permiten valorar el impacto en la seguridad del paciente y determinar los factores con mayor peso para la ocurrencia de eventos adversos relacionados con la medicación.

Es un estudio de tipo descriptivo, transversal, correlacional. La unidad de análisis son los procedimientos de administración de medicamentos intravenosos, que las enfermeras realizaron a los pacientes hospitalizados en los servicios de medicina interna, cardiología, neurología y gastroenterología de un Hospital Pediátrico de alta especialidad $(n=114)$, con el objeto de conocer el nivel de eficiencia y su relación con las variables intervinientes.

Los resultados obtenidos nos permitieron identificar que el índice de eficiencia general es de $85 \%$ y al compararlo con respecto al día de observación es estadísticamente significativo para el fin de semana.

Palabras claves: Calidad del Cuidado, seguridad del paciente, administración de medicamentos, evento adverso.

\section{Abstract}

The purpose of this study was to assess the quality of the intravenous drug management provided by nursing professionals. We used a specific instrument to measure the efficiency of this process. These estimates will hopefully allow us determine the impact of related factors and thus identify problems and establish improvement strategies.

This was a basic descriptive, cross-sectional and correlational study. The sample was formed out of 114 patients hos- pitalized in the internal medicine, cardiology, neurology, and gastroentherology units of a high specialty Pediatric Hospital. The results suggest that it is possible to estimate a related index of efficiency close to $85 \%$. We considered these findings as statistically significant.

Key words: quality of care, safety of the patient, drug management, adverse event. 


\section{INTRODUCCIÓN}

La seguridad del paciente, es un tema que ha destacado en los últimos años tanto a nivel nacional como internacional, ya que las cifras de muertes provocadas por errores médicos han aumentado en forma considerable. Según los reportes del Instituto de Medicina de los Estados Unidos ocurren entre 44000 y 98000 muertes anuales como resultado de errores médicos, ubicando este problema por arriba de los accidentes de motor, el cáncer de mama y el VIH/SIDA, lo que ha aumentado el costo nacional anual de los actos de atención de salud perjudiciales evitables entre 17000 y 29000 millones de dólares ${ }^{1,2,3,4}$. Por otra parte, en México esta temática ha sido investigada en diversas unidades hospitalarias como es el caso de Lavalle ${ }^{5}$ quien estudió la frecuencia y tipos de error en la prescripción de medicamentos en los expedientes, otro es el de Ruiz Piñeiro ${ }^{6}$ quien evaluó el nivel de eficiencia en la administración de medicamentos por vía oral.

Lo anterior nos lleva a reflexionar sobre uno de los aspectos primordiales que guían las acciones del profesional en salud, la cultura de la calidad. Uno de los expertos más reconocidos en este ámbito es Avedís Donabedian, quien considera que la calidad es la atención que se le proporcione al paciente, brindando el máximo y más completo bienestar en todo momento, logrando los mayores beneficios posibles y comprende tres dimensiones: La científico-técnica se refiere a la calidad real que el paciente recibe tras un diagnóstico o tratamiento. La interpersonal hace referencia a la forma en que se presta la asistencia sanitaria, así como en las expectativas y aspiraciones de los usuarios. Y el entorno, es el aspecto más íntimo de los lugares en los que se presta la atención el confort y las condiciones de accesibilidad ${ }^{7,8}$.

Además, Donabedian sistematizó la evaluación de la calidad de la asistencia sanitaria a partir de la tríada de estructura, proceso y resultado marcando la evolución conceptual y metodológica de la gestión de la calidad, que hoy en día es el paradigma dominante. Así la estructura se enfoca al análisis de la configuración física-organizativa, incluye los recursos humanos, materiales y financieros. Por lo que se refiere al proceso es el conjunto de actuaciones, decisiones y tareas encaminadas a la prevención, diagnóstico, tratamiento y rehabilitación de los usuarios. Por último, los resultados son los cambios en el paciente y en su estado de salud futuro, atribuibles a unos cuidados sanitarios ${ }^{8-11}$.
Por otra parte es importante destacar que dentro de la práctica del cuidado, los profesionales de enfermería realizan diversos procedimientos, por lo que deben poseer habilidades, destreza y conocimientos científicos para realizarlos de manera correcta, eficaz y segura. Sin embargo se debe tener en cuenta que dentro del entorno hospitalario existen diversos factores que intervienen tales como: número de personal, falta de conocimiento, sobrecarga de trabajo, sistemas de dotación, entre otras, que pueden ser causantes de errores que no eximen a las enfermeras de falla desde el punto de vista ético y jurídico, por tal motivo es necesario evaluar el cumplimiento de las normas institucionales en relación con la seguridad y establecer programas de mejora que incidan en la calidad de la atención de los pacientes.

En este sentido debemos reconocer entre las amenazas más comunes contra la seguridad de los pacientes pueden mencionarse los errores de medicación, por lo que se hace necesario identificar y reducir el número de estos y conocer sus factores de riesgo. Existen evidencias de que los niveles inadecuados de dotación de personal en los hospitales guardan relación con el aumento de errores de medicación, que pueden dar lugar a estancias hospitalarias más prolongadas o a índices más elevados de mortalidad. 1,12,13

Sin embargo para poder resolver el problema es importante conocer las causas, por lo que diversos investigadores tales como Tosta, De Bortoli, Bauer de Camargo, Inocenti, Ruiz Piñeiro, Fontan, Donoso, Aiken y sus colaboradores han descrito en estudios realizados en Brasil, México, Francia, Chile y Estados Unidos, las situaciones que conducen a los profesionales de enfermería a cometer errores en la administración de medicamentos destacando las fallas en el cumplimiento de políticas y procedimientos, del sistema de distribución y preparación, en la comunicación y conocimiento, en la prescripción y transcripción, la falta de atención, uso de abreviaturas, falta de un padrón de horarios, información no actualizada del paciente, no valorar el modo de infusión, además el no calcular las dosis o diluciones de manera correcta, o bien que el mismo profesional no prepare, administre y registre el medicamento, así como el nivel académico de los profesionales que administran la medicación ${ }^{6,14-21}$

Por todo lo anterior se decide realizar la investigación que tiene por objetivo Evaluar la calidad de la administración de medicamentos intravenosos, que realiza el profesional de enfermería de un Hospital Pediátrico de 
alta especialidad en la Cd. de México y su impacto en la seguridad del paciente.

\section{METODOLOGÍA}

Se realizó un estudio descriptivo, transversal, correlacional de enfoque cuantitativo y de diseño no experimental. La unidad de análisis fueron los 114 procedimientos de preparación y administración de medicamentos por vía intravenosa que aplicaron los profesionales de enfermería a los pacientes hospitalizados en los servicios de medicina interna, cardiología, neurología y gastroenterología, durante el período de recolección de datos, es decir del 15 de agosto al 15 de septiembre del 2007, de lunes a domingo.

\section{PROCEDIMIENTO}

Para la recolección de datos se efectuaron las observaciones de los procedimientos de preparación y administración de medicamentos por vía intravenosa, para lo cual se utilizó un instrumento de auditoría simultánea, el cual obtuvo una validación de contenido por medio de jueceo de expertos ${ }^{22}$. La validación se llevo a cabo mediante la emisión de la opinión de seis expertos en el procedimiento de preparación y administración de medicamentos, realizando un análisis de contenido de las observaciones realizadas por cada uno de ellos.

El instrumento consta de tres apartados, el primero describe los datos sociodemográficos de los profesionales de enfermería que realizaron el procedimiento (Edad, nivel académico, antigüedad en el servicio, turno y fecha de observación), el segundo contiene las actividades relevantes, de forma secuencial y correcta del procedimiento de administración de medicamentos y consta de 14 items y el tercero es un área para observaciones.

Cada uno de los ítems tiene un valor numérico el cual se asignó como "0" cuando no son llevadas a cabo o se cumplen de manera incorrecta y "1" a las actividades que se realizan de forma correcta, la suma total proporciona el nivel de eficiencia en el procedimiento por caso, actividad y la global. El índice de eficiencia por caso identifica a la persona que realiza cada procedimiento y así integrarlo al proceso de mejora continua, mientras que el índice de eficiencia por actividad permite detectar cuáles actividades se realizan con mayor y menor eficiencia, además de analizar las causas y la implementación de las estrategias de mejora. Por lo que respecta al índice de eficiencia global refleja el nivel de calidad con el cual se desarrolla el procedimiento y permite establecer las metas de mejora.

Estos índices representan el estándar de calidad del procedimiento, para lo cual existen niveles establecidos y validados $^{9}$ : Nivel de Excelencia alcanza entre 91 y $100 \%$, el significativo entre el 85 y el $90 \%$, el de cumplimiento parcial entre el 75 y el $84 \%$, cumplimiento mínimo entre el 70 y $74 \%$ y el de no cumplimiento por abajo del $70 \%$. Además se determino si existen diferencias significativas entre el nivel de eficiencia y el día en que se realizaron las observaciones, así como entre los niveles académicos.

La Muestra estuvo constituida por 114 procedimientos de administración de medicamentos intravenosos que realizaron 28 enfermeras profesionales, a quienes se observó durante las diversas etapas del proceso de preparación y administración de medicamentos intravenosos.

Dentro de las implicaciones éticas del estudio fue esencial destacar que se obtuvo el consentimiento informado a las autoridades de la institución y del departamento de enfermería, así como de las enfermeras y se garantizó el respeto en todo momento, la completa confidencialidad y el anonimato.

\section{ANÁLISIS DE DATOS}

Para el plan de análisis estadístico se elaboró una base de datos y se apoyó con un paquete estadístico Statistical Package for the Social Sciences (SPSS) versión 12.0.

El nivel de eficiencia en la administración de medicamentos por vía intravenosa se definió operacionalmente como el grado de cumplimiento en la realización del procedimiento con base a la norma establecida. La información se obtuvo de la observación directa no participante, hojas de atención de enfermería, indicaciones médicas y kárdex.

Por otra parte la seguridad del paciente se definió como el número de eventos adversos por medicamentos intravenosos que se presentaron en el servicio durante el tiempo de recolección de datos basados en los registros hospitalarios del programa de seguridad del paciente.

Sin embargo se registraron las variables sociodemográficas de Edad, nivel académico (Enfermera general, con postécnico, licenciada, especialista a nivel de posgrado), antigüedad en el servicio, turno (matutino, vespertino y nocturno) y fecha de observación (entre semana o fin de semana).

Al analizar la influencia de las variables sociodemográficas sobre el nivel de eficiencia en la administración de medicamentos se utilizaron las pruebas de comparación 
de T de student para muestras independientes y Análisis de varianza, así como de asociación de Pearson. Además se utilizaron pruebas estadísticas descriptivas como medidas de frecuencia, tendencia central y de dispersión.

\section{RESULTADOS}

Participaron en el estudio un total de 28 enfermeras, con los siguientes resultados de las variables sociodemográfica: una media de 33 años de edad $( \pm 7)$, el $50 \%$ tienen una formación académica de nivel licenciatura; con relación a la antigüedad dentro del servicio la media es de 21 meses ( \pm 25 ). Por lo que respecta al turno en que laboran el matutino destaca con un 39\%, el nocturno presentó un $36 \%$ y el vespertino un $25 \%$. Cabe mencionar que el $50 \%$ de las observaciones se realizaron entre semana y el otro $50 \%$ en fin de semana.

En lo relativo a las 114 evaluaciones realizadas de los procedimientos de administración de medicamentos intravenosos se observo que en promedio cada enfermera realizó 4 procedimientos ( \pm 1.6$)$. El índice de eficiencia global obtenido fue del $85 \%$ y el índice de eficiencia por caso fue: el $15 \%$ se encontró en un nivel de excelencia, en un nivel de cumplimiento significativo el $26 \%$, en un nivel de cumplimiento parcial el 55\% y finalmente el $4 \%$ en un nivel de cumplimiento mínimo.

En lo que respecta al índice de eficiencia por actividad fue de $85 \%$, donde los ítems que presentaron una mayor eficiencia dentro del procedimiento se refieren a si el profesional de enfermería revisa la indicación, se lava las manos, verifica las características del medicamento, comprueba la indicación frente a la etiqueta, prepara el medicamento con base a los cinco correctos, identifica al paciente y realiza los registros en la hoja de enfermería se encontraron entre el 99 y $100 \%$. Con relación a los ítems con una eficiencia media (84 al 94\%), los cuales indican si la enfermera prepara el medicamento en la hora correcta y lo identifica. Mientras que los ítems con menor eficiencia (29 a 77\%) son los que describen si los profesionales verifican la fecha de caducidad, valoran la presencia de un acceso venoso permeable, informan al familiar sobre el procedimiento, administran el medicamento en el horario indicado, valoran que la velocidad de administración sea correcta y evalúan la presencia de reacciones (Tabla 1).

Por otra parte se determino si hay diferencias entre el nivel de eficiencia y el día de la semana en que se realizaron las observaciones, encontrándose diferencias significativas ( $p=0.019$ ). Es decir se observa que la eficiencia en fin de semana tuvo un promedio más alto (86.34), al compararlo con las observaciones realizadas entre semana (83.81) (gráfica 1).

Sin embargo, no se encontró una diferencia estadísticamente significativa entre el nivel de eficiencia en la administración de medicamentos y las variables sociodemográficas de turno laboral $(p=0.171)$ y nivel académi-

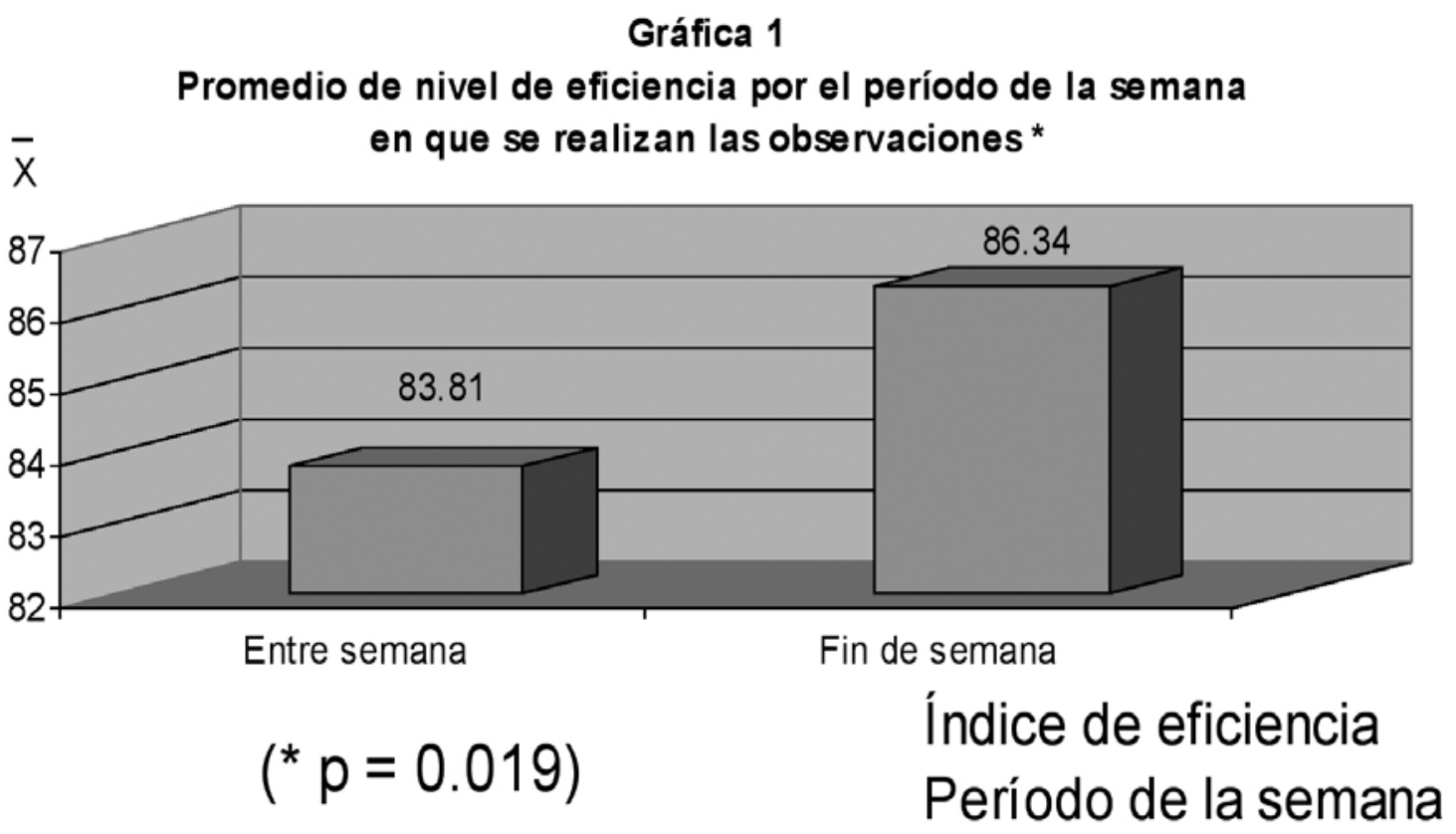

Fuente: 114 observaciones sobre el nivel de eficiencia en la administración de medicamentos por vía intravenosa 


\section{Tabla 1}

Índice de eficiencia por actividad del procedimiento de administración de medicamentos por vía intravenosa

Actividades

1. Revisa la hoja de indicaciones médicas, el kardex, tarjeta u hoja de enfermería donde se encuentra la indicación para la administración del medicamento

2. Se lava las manos
Fx

$\%$

5. Comprueba la tarjeta, kardex, hoja de enfermería o de indicaciones frente a la etiqueta del medicamento 
co $(p=0.154)$. Por otra parte se encontró que no existe una asociación lineal entre el nivel de eficiencia y la antigüedad del personal de enfermería en el servicio ( $p=$ 0.320).

Dentro de la valoración de eventos adversos se localizaron los reportes de tres incidentes, el 67\% se presentaron durante el período de la recolección de datos, predominando el turno vespertino y el nivel académico fue enfermeras generales. Lo referente a las condiciones de los eventos adversos identificados fueron en dos terceras partes por administrar el medicamento a paciente equivocado y la tercera parte restante fue porque al transcribir la indicación médica esta se realizó en el kardex incorrecto.

\section{DISCUSIÓN}

En términos generales se analizaron los resultados obtenidos, donde se observo la actuación del profesional de enfermería durante el procedimiento de administración de medicamentos por vía intravenosa, encontrando una asociación con las investigaciones realizadas por Inocenti Miasso ${ }^{15,16}$ y Tosta $^{14}$, quienes identificaron cuatro causas por las que se presentan los eventos y son falla en el cumplimiento de políticas y procedimientos, en el sistema de distribución y preparación de los medicamentos, en la comunicación y en el conocimiento.

Otro aspecto que se destacó es que no se valora la velocidad de administración, es decir que no se tiene el cuidado adecuado con el volumen y el tiempo de infusión en que deben administrarse los fármacos, relacionado con la falta de conocimiento o distracción, o por sobrecarga de trabajo. Lo que concuerda con la investigación realizada por Donoso y Fuentes ${ }^{20}$. Sin embargo, se identificaron otros factores como que el personal que prepara el medicamento no es el mismo que lo administra o lo registra, los cuales son elementos que favorecen la presencia de eventos adversos, como lo muestra también el estudio de Fontan, Mille y Brion ${ }^{18}$.

En lo referente al índice de eficiencia obtenido y en relación al turno laboral, aún cuando no hay diferencias significativas el turno nocturno presentó un mejor nivel de calidad, esto concuerda con lo identificado por Lavalle ${ }^{22}$. Por otra parte el índice de eficiencia del procedimiento con relación al nivel académico de las enfermeras se encontró que a mayor grado de estudios presentan un mejor nivel de calidad en sus procedimientos medida por el índice de cumplimiento. Esto coincide con la investigación realizada por Aiken ${ }^{21}$ quien encontró que por cada 10\% de aumento en la proporción de enfermeras con grados superiores (licenciatura), disminuye el riesgo de mortalidad de los pacientes en un $5 \%$ en servicios hospitalarios.

Por otra parte, el índice de eficiencia que se encontró es en el nivel significativo, el cual resulta aceptable, dado que el personal cumple con la mayoría de los requisitos del proceso y alcanza un estándar de calidad del $85 \%$. Situación que nos permite a partir de conocerlo, evaluar, controlar y establecer programas para la mejora de la calidad del cuidado enfermero.

Se destaca en el estudio que no se cuenta con una cultura del reporte de eventos adversos. Sin embargo esta es una estrategia que ha sido descrita en diversas investigaciones tal es el caso de Inocienti ${ }^{15,16}$, Bauer de Camargo ${ }^{19}$, Rodrigo ${ }^{1}$, De Bortoli ${ }^{20}$ y Donoso ${ }^{17}$. Por lo que se deben establecer programas que lo apoyen de forma anónima y no punitiva, con el fin de implantar sistemas de notificación voluntaria y concientización del personal, ya que el reconocimiento de estos es el primer paso en su prevención. ${ }^{13}$

Existen algunas otras estrategias para mejorar la calidad y la seguridad en la atención a los pacientes según reportes de investigación como el de Inocenti Miasso ${ }^{15,16}$, Rodrigo ${ }^{1}$, Fontan, Mille y Brion ${ }^{18}$ quienes destaca contar con normas para pacientes pediátricos, formación de una comisión multidisciplinaria para evaluar el sistema de administración de medicamentos, grupos de discusión, capacitación continua y motivación del personal.

\section{CONCLUSIONES Y RECOMENDACIONES}

La calidad es un atributo que debe ser propiciada y evaluada en los cuidados que proporcionan los profesionales de enfermería situación que permite garantizar que los servicios de salud sean eficientes. Evaluar la calidad del cuidado implica monitoreo, seguimiento, medición en la ejecución y realizar comparaciones de las actividades profesionales del personal y posteriormente con base en los resultados diseñar estrategias de mejora que permitan brindar cuidados seguros a los pacientes.

Para lo cual es que los profesionales de enfermería así como las dirigentes analicen los errores o desviaciones en la medicación y sus causas; identificar los medicamentos o categorías de medicamentos con los que resulta fácil cometer errores, e introducir mejoras para evitar que ocurran o que su número disminuya.

Los resultados que aquí se presentan describen el fenómeno en particular de la población estudiada, sin embargo las estrategias que se recomiendan pueden ser 


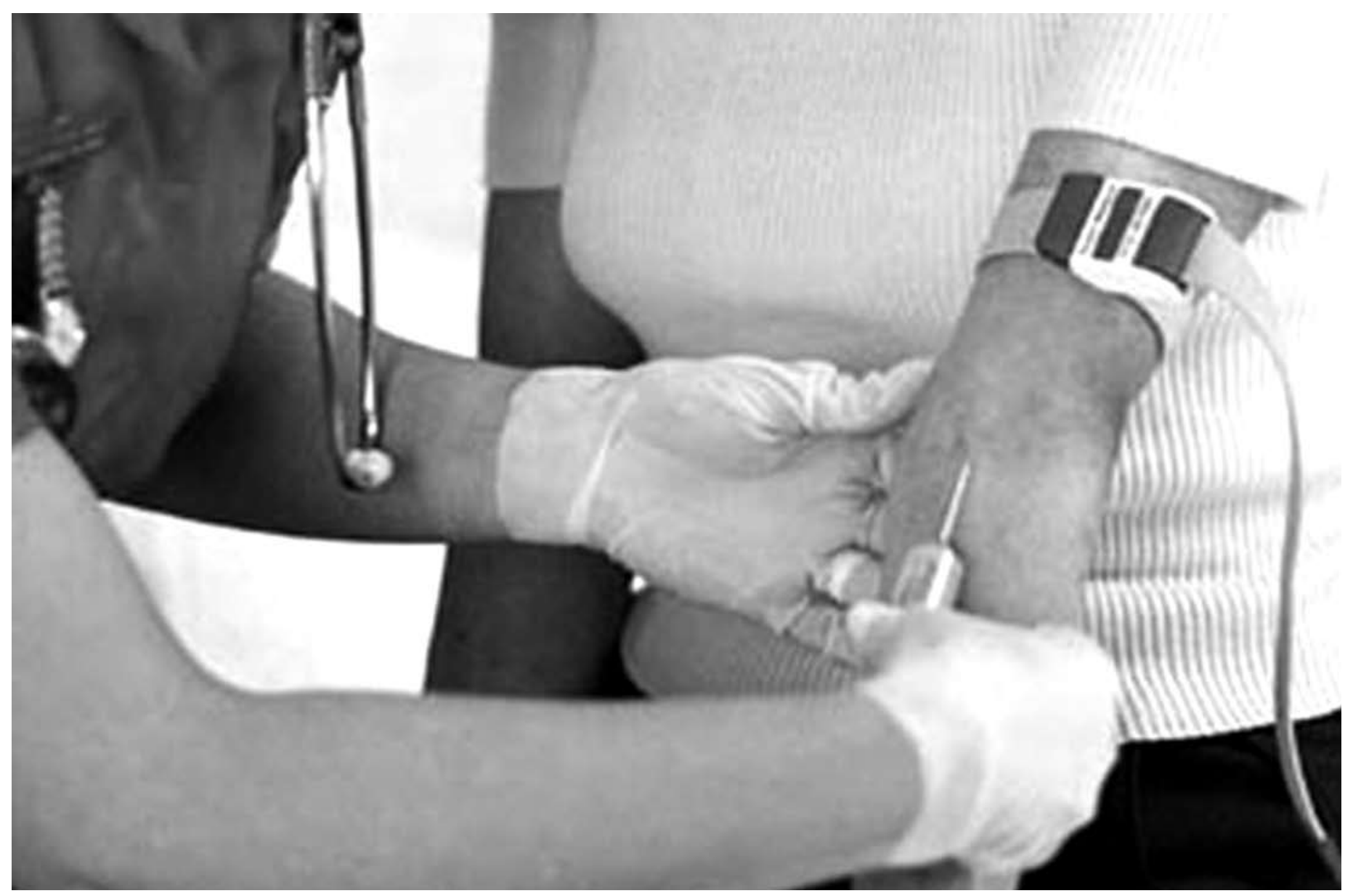

aplicadas en otras poblaciones. Pero también se pueden hacer replicas de este estudio en otros servicios de la misma institución o bien en otras, instituciones a fin de comparar los resultados.

Dentro de las recomendaciones que se.plantean son: a) Se debe evitar sacar los medicamentos de los empaques originales lo que conlleva a tener un mayor control de la caducidad de éstos; b) Los profesionales que preparan los fármacos deben ser los mismos que los administren y registren; c) Se preparen en los horarios indicados para evitar que se pierdan sus propiedades o se contaminen. Por otra parte es necesario buscar estrategias de capacitación para lograr el dominio de las diluciones correctas de los medicamentos, tiempos de infusión e identificación de reacciones adversas, ya que todos estos, son aspectos trascendentes para evitar la presencia de eventos adversos. Otro aspecto que debe cuidarse es involucrar al paciente y su familia y/o el paciente en la medicación a fin de obtener información adecuada sobre medicamentos que el paciente toma con frecuencia, alergias $u$ otros tratamientos que pueden contribuir a la presencia de eventos adversos. Finalmente es indispensable hacer conciencia en los profesionales de Enfermería.de la responsabilidad de cumplir con las normas en la administración de medicamentos los "cinco correctos" y evitar con ello riesgos innecesarios a los pacientes que pueden en algunos casos ocasionar consecuencias graves. Así también realizar estudios que nos permitan.identificar elementos relacionados con la gestión, la disposición y distribución de los medicamentos así como la organización de los servicios como fuentes documentadas que favorecen los errores en la medicación.

\section{REFERENCIAS.BIBLIOGRÁFICAS}

1 Rodrigo M, Beltrán I, Berradre E, Alonso A, Marcotegui F.

Trabajando por la seguridad de los pacientes: puesta en marcha de un grupo de trabajo sobre errores de medicación. Revista Anales del Sistema Sanitario de Navarra [serie en Internet]. 2004 Enero-Abril [acceso 19 Octubre 2005]; 27 (1): [aprox. 8 p.]. Disponible en: http://www. Cfna varra.es/SALUD/ANALES/textos/vol27/n1/cartas 1.html.

2 Consejo Internacional de Enfermeras [La enfermería importa-seguridad de los pacientes] Hoja de datos de la 
Alianza Mundial de Profesiones de Salud. Comunicado de Prensa 29 de abril de 2002 Los profesionales de salud piden prioridad para la seguridad del paciente.[acceso 8 Junio 2006]. Disponible en: http://www.icn.ch/ matters_ptsafetysp.htm.

3 Franco A, La seguridad clínica de los pacientes: entendiendo el problema. Colombia Médica. 2005; 36 (2): 130-133.

4 Rivero SO, Paredes SR, Durante MI, Rivero WP, Kifshitz A, García PE. et al. Los errores médicos. En Ética en el Ejerció de la Medicina. $1^{\text {a }}$. ed. México: Editorial Médica Panamericana; 2006..p. 151 - 162.

5 Lavalle VA, Payro CT, Martínez CK, Torres NP, Hernández DL y Flores NG. El error médico en la prescripción de medicamentos y el impacto de una intervención educativa. Bol Med del Hosp Infant Mex. 2007 Marzo-Abril; 64: p. $83-90$.

6 Ruiz PR, Montesinos JG, Carlón CM, Vega HS y Barrera SM. Nivel de eficiencia en administración de medicamentos por vía oral. Revista Mexicana de Enfermería Cardiológico. 2002 Septiembre-Diciembre; 10 (3): p. 90 - 95.

7 Donabedian A. La calidad de la atención médica: Definición y métodos de evaluación. Universidad de Michigan, EUA: Ediciones Científicas La Prensa Medica Mexicana S.A; 1984. p. 194.

8 Ayuso MD y Grande SR. Gestión de la calidad. En La gestión de enfermería y los servicios generales en las organizaciones sanitarias. $1^{\text {a }}$. ed. España: Editorial Diaz de Santos; 2006. p. 527- 557.

9 Ortega VM, Suárez VM, Jiménez VM, Añorve GA, Cruz CM, Cruz AG. et al..Elementos conceptuales de la calidad. En Manual de evaluación del servicio de calidad en enfermería. Estrategias para su aplicación. 1ª. ed. México: Interamericana; 2006. p. 1-15.

10 Salvadores FP, Sánchez LF y Jiménez FR. Evaluación de la calidad. En Manual de administración de los servicios de enfermería. $1^{\text {a }}$. ed. Barcelona: Ariel Ciencias Médicas; 2002. p. $243-256$.

11 Síntesis ejecutiva - Seguridad del paciente [base de datos en Internet] [citado 19 Octubre 2005]. Disponible en: http://www. salud.gob.mx/.../evaluación/publicaciones/ síntesis/síntesis 13.pdf.

12 Consejo Internacional de Enfermeras. Personas fiables, vidas salvadas..Anexo 8: Seguridad de los pacientes. Ginebra, Suiza; 2006.

13 Simpson JH, y cols. Reduciendo errores de medicamentos en Unidad de Cuidados Intensivo Neonatal. Archi- ves of disease in Childhood Fetal and Neonatal Edition [serie en internet]. 2004 Noviembre [acceso 18 Octubre 2005]; 89 (6): [aprox. 6 p.]. Disponible en:http://www. Prema turos.cl/webdicembre/enfermerianeonatal/erroresmedicamentos. htm.

14 Tosta CV y Bortoli CS. Errores en la administración de medicamentos: análisis de situaciones relatadas por los profesionales de enfermería. Investigación y Educación en enfermería. 2001 Septiembre; XIX (2):.p. 26-35.

15 Inocenti MA, Bauer CS, De Bortoli CS, Grau CR, Oliveira RC y Trevisan FF. O processo de preparo e administração de medicamentos. Identificação de problemas para propor melhorias e prevenir erros de medicação. Rev. Latino-am Enfermagem. 2006 Mayo - Junio; 14 (4): p. 354 - 63.

16 Inocenti MA, Renata GC, De Bortoli CS, Bauer CS y Trevisan FF. Erros de medicaçãs: Tipos, fatores causais e providências tomadas em quatro hospitais brasileiros. Rev. Esc. Enferm USP. 2006; 40 (4): p. 524 - 32.

17 Donoso FA y Fuetes RI. Eventos adversos en UCl. Rev Chil Pediatr. 2004; 75 (3): p. 233 - 239.

18 Fontan JE, Mille Fy Brion F. La administración de medicamentos al niño hospitalizado. Archives de Pédiatrie [serie en Internet]. 2004 Octubre [acceso 18 Octubre 2005]; 11(10): [aprox. 3 p.]. Disponible en: http://www. siicsalud. com/td/pediatria/resiicpediatria85.htm.

19 Bauer CS y De Bortoli CS. Erros de medicação em Hospital Universitário: Tipo, causa, sugestões e providências. Rev. Bras Enferm, 2004 Noviembre - Diciembre; 57 (6): p. $671-4$.

20 De Bortoli CS, Cardos AT, Perufo OS y Hincares JC. O sistema de medicação nos hospitais e sua avaliação por um grupo de profissionais. Rev. Esc. Enferm USP. 2005; 39 (3): p. $280-7$.

21 Aiken LH, Clarke SP, Cheung RB, Sloane DM y Silber JH. Educational Levels of Hospital Nurses and Surgical Patient Mortality. JAMA. 2003 September; 290 (12): p.1617 $-1623$

22 Polit DF y Hungler BP. Evaluación de la calidad de los datos. En Investigación científica en ciencias de la saIud. 6ª . ed. México: McGraw-Hill Interamericana; 2000. p. 399-401.

\section{DIRECCIÓN PARA CORRESPONDENCIA}

Mtra. Patricia Adriana Cárdenas Sánchez

p_adriana1975@yahoo.com.mx 